EESTI NSV TEADUSTE AKADEEMIA TOIMETISED.

FOOSIKA * MATEMAATIKA

ИЗВЕСТИЯ АКАДЕМИИ НАУК ЭСТОНСКОИ ССР. ФИЗИКА * МАТЕМАТИКА

PROCEEDINGS OF THE ACADEMY OF SCIENCES OF THE ESTONIAN SSR.

PHYSICS * MATHEMATICS

$1988,37,1$

УдК 539.12

Р.-К. ЛОЙДЕ

\title{
К ТЕОРИИ БЕЗМАССОВЫХ КАЛИБРОВОЧНЫХ ПОЛЕЙ
}

\author{
(Представил Х. Керес)
}

Рассмотрены безмассовые волновые уравнения в формализме ковариантных проекционных операторов спина. Приведены необходимые и достаточные условия калибровочной инвариантности уравнения и ограничения на внешний источник.

\section{1. Введение}

Важную роль в современной теории элементарных частиц играют векторные калибровочные поля. Суперсимметрия и супергравитация требуют существования калибровочных полей с более высокими спиральностями [ $\left.{ }^{1}\right]$. Поля гравитона и гравитино со спиральностями 2 и $3 / 2$ входят в один безмассовый супермультиплет. Суперсимметрия допускает для гравитона партнера со спиральностью 5/2, но пока еще соответствующая теория - гипергравитация - связана с трудностями. Что касается математического исследования калибровочных полей с высокими спиральностями, то здесь еще полная теория отсутствует. В работах $\left[{ }^{2,3}\right]$, где были рассмотрены некоторые уравнения для высших спинов, авторы пришли к выводу, что принципы построения массивных уравнений не применимы в случае безмассовых калибровочно-инварнантных уравнений. В данной работе мы покажем, что это не так, и выведем общие условия на матрицы уравнения в безмассовом случае.

Для полей со спиральностями $3 / 2$ и $5 / 2$ нами было показано, что принципы построения массивных уравнений применимы и в безмассовом случае $\left[{ }^{4,5}\right]$. При этом условия, налагаемые на матрицы уравнения, существенно отличаются от условий в массивном случае. В безмассовом случае определенные миноры спин-блоков должны равняться нулю. Последнее условие слабее, чем требование нильпотентности спинблоков в массивном случае. Соответственно для описания безмассовых полей размерность представления может быть меньше. Для описания спина $5 / 2$ с массой $m$ требуется, например, симметричное тензор-биспинорное поле $\psi_{\alpha}^{\mu v}$ и биспинорное поле $\lambda_{\alpha}$, а для описания соответствующей спиральности в безмассовом случае достаточно поля $\psi \alpha^{\mu v}$.

Мы исходим из допущения, что калибровочно-инвариантные уразнения получаются из соответствующих массивных уравнений. Приведены необходимые и достаточные условия для калибровочной инвариантности уравнений при $m=0$ и ограничения на внешний источник. Мы применяем формализм ковариантных проекционных операторов, развитый в $\left[{ }^{6,7}\right]$.

\section{2. Массивные уравнения}

Приведем некоторые необходимые понятия из теории массивных релятивистски-инвариантных волновых уравнений в формализме проекционных операторов спина $\left[{ }^{6,7}\right]$. 
Рассмотрим уравнение порядка $n$

$$
\pi \Psi=m^{n} \Psi \text {. }
$$

Допустим, что $\Psi$ разложено в прямую сумму $r$ неприводимых полей $\psi_{i}$, которые преобразуются по представлениям $\left(k_{i}, l_{i}\right)$ группы Лоренца. Тогда оператор $\pi$ представляется в блочном виде

$$
\pi=(-\square)^{n / 2}\left|a_{i j} P_{i j}\right|,
$$

где $a_{i j}$ произвольные постоянные, конкретный выбор которых определяет свойства уравнения. Операторы $P_{i j}$ являются линейными комбинациями ковариантных операторов проектирования спина $P_{i j}^{s}$

$$
P_{i j}=\sum_{s} \beta_{i j}(s) P_{i j}^{s} .
$$

Общие выражения для вычисления коэффициентов $\beta_{i j}(s)$ при произвольном $s$ приведены в $\left[{ }^{8}\right]$. В случае уравнений первого порядка коэффициенты $\beta_{i j}(s)$ хорошо известны (см., напр., $\left[{ }^{6}\right]$ ).

Свойства уравнения определяются приведенными матрицами $\beta_{s}$ спина $s$

$$
\beta_{s}=\left|a_{i j} \beta_{i j}(s)\right| .
$$

Матрицы $\beta_{s}$ являются квадратичными $r \times r$-матрицами. Ненулевые собственные значения $\lambda_{s}$ определяют массы состояний, соответствуюших спину $s: m_{s}=m \lambda_{s}^{-1 / n}$. В случае, когда спин $s$ данным уравнением не описывается, марица $\beta_{s}$ должна быть нильпотентной, т. е. $\left(\beta_{s}\right)^{a}=0$, где $a>1$.

Так как не все поля $\psi_{i}$ в разложении $\Psi$ содержат спин $s$, в дальнейшем нам удобнее работать со спин-блоками $\tilde{\beta}_{s}$, которые получаются из $\beta_{s}$ исключением тех рядов и столбцов, которые соответствуют представлениям, несодержащим спин $s$ (элементы этих рядов и столбцов равны нулю). Матрицы $\tilde{\beta}_{s}$, соответствуют спин-блокам Гельфанда-Яглома. Допустим, что первые $r_{s} \leqslant r$ представлений содержат спин $s$. Тогда

$$
\tilde{\beta}_{s}=\left|a_{i j} \beta_{i j}(s)\right|, \quad i, j=1,2, \ldots, r_{s}
$$

является квадратичной $r_{s} \times r_{s}$-матрицей.

\section{3. Безмассовые уравнения}

Рассмотрим вместо (1) безмассовое уравнение порядка $n$

$$
\pi \Psi=0 \text {. }
$$

Выведем необходимые и достаточные условия для калибровочной инвариантности уравнения (6) относительно калибровочного преобразования

$$
\Psi \rightarrow \Psi+Q^{g}
$$

и существования оператора $Q^{z}$ со свойством

$$
Q^{z} \pi=0 .
$$

В случае уравнения с источником $\pi \Psi=J$ оператор $Q^{z}$ дает ограничение на источник $Q^{z} J=0$. Последнее условие означает, что допустимы только такие взаимодействия, которые дают источники, удовлетворяющие $Q^{z} J=0$.

Допустим, что операторы $Q^{g}$ и $Q^{z}$ являются дифференциальными 
операторами первого порядка, как это общепринято. В принципе, можно рассматривать и калибровочные преобразования с производными более высокого порядка. Но поскольку калибровочная свобода необходима для элиминирования ненужных спиральностей, то фиксирование калибровки привело бы к уравнениям выше второго порядка. Такие возможности еще до конца не исследованы, хотя и в [ $\left.{ }^{9}\right]$ допускается применение уравнений выше второго порядка.

Рассмотрим сперва инвариантность уравнения (6) относительно калибровочного преобразования (7). Разложим поле $\varepsilon$ в прямую сумму неприводимых полей $\varepsilon_{k}$. Тогда для калибровочной инвариантности уравнения надо рассматривать инвариантность отдельно для каждого поля $\varepsilon_{k}$. При этом в калибровочное преобразование входят только такие поля $\varepsilon_{k}$, которые зацепляются с представлениями $i$ в разложении $\Psi$, т. е. $\left(k_{k}, l_{k}\right) \otimes(1 / 2,1 / 2)$ содержит неприводимые представления, входящие в разложение $\Psi=\psi_{1} \oplus \ldots \oplus \psi_{r}$. Перепишем (7) для поля $\varepsilon_{k}$ в виде

$$
\psi_{i} \rightarrow \psi_{i}+Q_{i k}^{g} \varepsilon_{k}, \quad i=1,2, \ldots, r .
$$

Так как $Q^{g}$ является дифференциальным оператором первого порядка, то оно выражается через проекционные операторы $P_{i k^{8}}$ в следующем общем виде

$$
Q_{i k}^{g}=a_{i} \sum_{s} \alpha_{i k}(s)\left(\sqrt{\square} P_{i k}^{s}\right),
$$

где $a_{i}-$ коэффициенты, подлежащие определению, а $\alpha_{i k}(s)$ однозначно определены (см. формулу (2.8) в $\left.\left[{ }^{6}\right]\right)$.

Допустим, что представление $k$ зацепляется с $r_{g}$ представлениями $i$. Без потери общности можно считать, что этими являются представления $i=1,2, \ldots, r_{g}$. Следовательно, в (9) $Q_{i k^{g}} \neq 0$, если $i=1,2, \ldots$ $\ldots, r_{g}$ и $Q_{i k} g=0$, если $i=r_{g}+1, \ldots, r_{s}$. Используя свойство проекционных операторов - $P_{i j}^{s} P_{j k}^{s^{\prime}}=\delta_{s s^{\prime}} P_{i k}^{s}$, можно показать, что условие калибровочной инвариантности $\pi Q^{g}=0$ приводится для каждого спина $s$ в разложении (10) к следующему алгебраическому уравнению

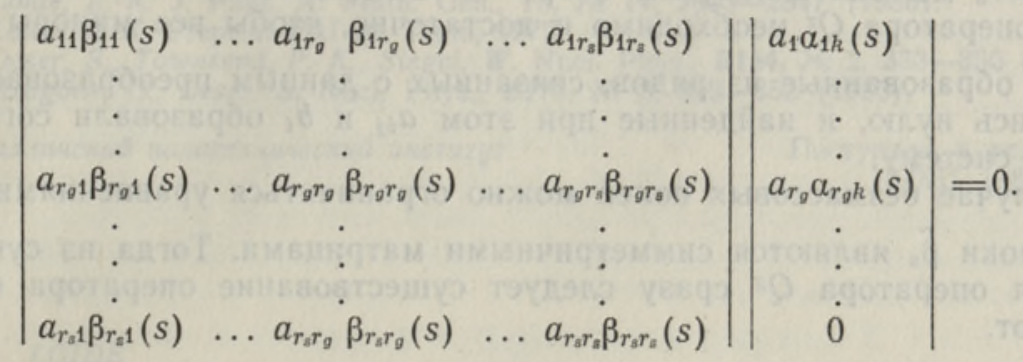

Мы получили систему линейных однородных уравнений относительно $a_{i} \alpha_{i k}(s)$, которые определяют $a_{i}\left(i=1,2, \ldots, r_{g}\right)$. Данная система имеет нетривиальные решения для $a_{i}$ тогда и только тогда, когда все $r_{g} \times r_{g}$ миноры $M$ матрицы $\tilde{\beta}_{s}$, образованные из первых $r_{g}$ столбцов, равняются нулю. В частном случае $r_{g}=r_{s}$ получим просто $\operatorname{det} \tilde{\beta}_{s}=0$. Данное условие налагает ограничения на коэффициенты уравнения $a_{i j}\left(i=1, \ldots, r_{s} ; j=1, \ldots, r_{g}\right)$. Условие равенства нулю всех миноров $M$, образованных из $r_{g}$ столбцов, связанных с данным калибровочным преобразованием, является только необходимым условием калибровочной инвариантности уравнения (6). Так как $a_{i j}$ и $a_{i}$ от спина $s$ не зависят, то надо еще требовать согласованности найденных $a_{i j}$ и $a_{i}$ для всех спинов $s$ в калибровочном преобразовании (9), и всех по- 
лей $\varepsilon_{k}$. Следовательно, для калиб̆ровочной инвариантности уравнения (6) относительно поля $\varepsilon_{k}$ необходимо и достаточно, чтобы все миноры матриц $\tilde{\beta}_{s}$, образованные из столбцов, связанных с калибровочным преобразованием, равнялись нулю, и найденные при этом $a_{i j}$ и $a_{i}$ образовали согласованную систему.

Из вышеприведенного видно, что условия для определения блоков Гельфанда-Яглома в случае безмассовых уравнений отличаются от соответствующих условий в случае массивных уравнений, хотя уравнение (6) получается из уравнения (1) подставкой $m=0$.

Теперь рассмотрим существование оператора $Q^{z}$ со свойством $Q^{z} \pi=0$, который дает ограничение на источник поля. Если уравнение инвариантно относительно калибровочного преобразования, то это означает, что выбором калибровки можно некоторые спины из уравнения элиминировать. Соответствующее ограничение должно быть наложено и на источник поля. Когда уравнение инвариантно относительно калибровочного преобразования с представлением $\varepsilon_{k}$, данного операторами $P_{i k}{ }^{s}$, то необходимо требовать существования оператора

$$
Q^{z}=\left|Q_{k i}^{z}\right|, \quad i=1,2, \ldots, r,
$$

где $Q_{k i} i^{2}$ - линейные дифференциальные операторы вида

$$
Q_{k i}^{z}=b_{i} \sum_{s} \alpha_{k i}(s)\left(\sqrt{\square} P_{k i}^{s}\right)
$$

и $b_{i}$ - коэффициенты, подлежащие определению. Требуя $Q^{z} \pi=0$, мы аналогично (11) получим следующее алгебраическое уравнение

$$
\left|b_{1} \alpha_{k 1}(s) \ldots b_{r_{g}} \alpha_{k r_{g}}(s) \ldots 0\right| \tilde{\beta}_{s}=0
$$

Полученная система относительно $b_{i} \alpha_{k i}(s)\left(i=1,2, \ldots, r_{g}\right)$ имеет нетривиальные решения при условии, что все $r_{g} \times r_{g}$ миноры $M$ матрицы $\tilde{\beta}_{s}$, образованные из первых $r_{g}$ рядов, равняются нулю. В частном случае $r_{g}=r_{s}$ мы опять получим $\operatorname{det} \tilde{\beta}_{s}=0$. Следовательно, для существования оператора $Q^{z}$ необходимо и достаточно, чтобы все миноры мат-

риц $\tilde{\beta}_{s}$, образованные из рядов, связанных с данным преобразованием, равнялись нулю, и найденные при этом $a_{i j}$ и $b_{i}$ образовали согласованную систему.

В случае безмассовых полей можно ограничиться уравнениями, где спин-блоки $\tilde{\beta}_{s}$ являются симметричными матрицами. Тогда из существования оператора $Q^{g}$ сразу следует существование оператора $Q^{z}$, и наоборот.

\section{4. Выводы}

Итак, приведены необходимые и достаточные условия для калибровочной инвариантности безмассовых полей и существования ограничений на источник. Калибровочная инвариантность означает существование оператора $Q^{g}$ со свойством $\pi Q^{g}=0$, ограничение на источник связано с оператором $Q^{z}$ со свойством $Q^{z} \pi=0$. Мы рассмотрели только уравнения. Что касается лагранжианов, то они выводятся, как и в массивном случае, из уравнения, выбрав соответствующую инвариантную билинейную форму.

Безмассовые уравнения для спиральности $3 / 2$ были исследованы в $\left[{ }^{4}\right]$. Оказалось, что для вектор-биспинорного поля существует целый 
класс безмассовых уравнений, которые отличаются тем, что соответствующие массивные уравнения отличаются спектром масс. Калибровочная инвариантность и ограничения на источник следуют из условий $\operatorname{det} \tilde{\beta}_{1 / 2}=0$. Безмассовые уравнения для спиральности 2 следуют из массивных уравнений для симметричного тензорного поля, рассмотренных в $\left[{ }^{7}\right]$, при условии $\operatorname{det} \tilde{\beta}_{0}=0$.

Более интересным является случай спиральности 5/2. Для описания безмассового поля достаточно симметричного тензор-биспинора. Калибровочное преобразование при этом дается с помощью вектор-биспинора. Оказывается, что калибровочное преобразование связано только с представлением $(1,1 / 2) \oplus(1 / 2,1)$ из вектор-биспинора, где компоненты, соответствующие биспинору, отсутствуют [ $\left.{ }^{5}\right]$. Необходимым и достаточным условием калибровочной инвариантности является $\operatorname{det} \tilde{\beta}_{3 / 2}=\operatorname{det} \tilde{\beta}_{1 / 2}=0$. Когда для спиральности $5 / 2$ применяются симметричное тензор-биспинорное поле и биспинорное поле, то требуются общие условия на миноры матриц $\tilde{\beta}_{3 / 2}$ и $\tilde{\beta}_{1 / 2}$. Одно такое уравнение рассмотрено в $\left[{ }^{5}\right]$. В [ $\left.{ }^{10}\right]$ описана спиральность $5 / 2$ с помощью специального несимметричного тензор-биспинорного поля, которое называется тетрадоподобным полем. Общий алгебраический анализ показывает, что уравнения для тетрадоподобного поля эквивалентны уравнениям для симметричного тензор-биспинорного поля,

\section{Л И ТЕ Р А Т У Р А}

1. Van Nienwenhuizen, P. Phys. Rep., 68, № 4, 189-398 (1981).

2. Berends, F. A., van Holten, J. W., van Nienwenhuizen, P., de Wit, P. Nucl. Phys., B154, № 2, 261-282 (1979).

3. Berends, F. A., van Reisen, J. C. J. M. Nucl. Phys., B164, № 2, 286-302 (1980).

4. Loide, R.-K., Polt, A. ENSV TA Toim. Füüs. Matem., 35, № 1, 43-55 (1986).

5. Loide, R.-K. J. Phys. A: Math. Gen., 19, № 5, 811-820 (1986).

6. Loide, R.-K. J. Phys. A: Math. Gen., 17, № 12, 2535-2550 (1984).

7. Loide, R.-K. J. Phys. A: Math. Gen., 18, № 14, 2833-2847 (1985).

8. Loide, R.-K. Preprint FAI-10. Tartu, 1972.

9. Deser, S., Townsend, P. K., Siegel, W. Nucl. Phys., B184, № 2, 333-350 (1981).

10. Aragone, C., Deser, S. Nucl. Phys., B170, № 2, 329-352 (1980).

Таллинский политехнический институт

Поступила в редакцию 18/XII 1987

\section{R.-K. LOIDE}

\section{NULLMASSIGA KALIBRATSIOONVÄLJADE TEOORIAST}

Artiklis on käsitletud massita lainevōrrandeid kovariantsete spinniprojektsioonioperaa. torite formalismis ning antud tarvilikud ja piisavad tingimused võrrandi kalibratsioon. invariantsuseks ja allikatingimused välisele allikale.

\section{R.-K. LOIDE}

\section{TO THE THEORY OF MASSLESS GAUGE FIELDS}

In the given paper the massless wave equations in the formalism of covariant spinprojection operators are treated. Necessary and sufficient conditions for a gauge invariance of a given equation and the source constraints to an external source are presented. 